\title{
THE CLINICAL APPLICATION OF EXTERNALLY POWERED ARTIFICIAL ARMS
}

\author{
D. S. McKenziE, London, England
}

\section{THE CONVENTIONAL PROSTHESIS}

Before considering the introduction of external powering and sophisticated control systems to artificial arms a review of the functional attainments of those equipped with hitherto conventional arms would be appropriate. It is hoped that the following description of the current British conventional arms will help to put matters in perspective.

The below-elbow prosthesis-The socket, which is fitted to a cast of the stump, terminates in a mechanism which permits ready attachment and detachment of the hand, or other terminal device, on the snap-catch principle. This mechanism also allows the terminal device to be rotated passively about the long axis of the arm to simulate pronation and supination, spring-loaded balls engaging with holes on the base of the terminal device so that the position can be regulated in 30-degree increments. This movement can also be locked positively by a bolt. The most popular hand is made with the fingers crooked in such a way that a brief case or similar object can be carried. The thumb is articulated at the base and spring loaded so that it will oppose the side of the index finger, or alternatively the tips of the index and middle fingers. Provision can be made for the thumb to be actively movable. Articulated fingers which can be placed in position passively are popular with some patients for cosmetic reasons, while others feel that this reduces the functional value of the hand. The arm is suspended by a webbing harness, the main component of which passes in front of the ipsilateral shoulder and across the back to loop round the contralateral axilla. A strap also passes immediately above the olecranon on the amputated side to hold the prosthesis on the stump when the elbow is flexed. The harness also provides a fixation point posterior to the contralateral axilla for the operation cord, which passes from this point across the back down to the arm to provide active movement for the terminal device. Rounding of the shoulders, flexion of the shoulder joint on the amputated side or extension of the elbow or any combination of the three causes this cord to retract from the terminal device and so provides a pull. A travel of three inches can usually be obtained by forearm amputees, and as the large muscle groups round the shoulders are thus harnessed, very considerable power is available.

The above-elbow prosthesis-The forearm of the above-elbow prosthesis is essentially similar to the above. The elbow joint mechanism incorporates a lock which enables the wearer to select the angle of elbow flexion in a number of alternative positions. The lock is usually operated by a reciprocating mechanism such that the first pull on its operating cord causes the lock to disengage and the next pull to engage, etc. A turntable is incorporated immediately above the lock mechanism to permit medial and lateral rotation about the long axis of the upper arm, thus simulating rotation at the shoulder. This, like the wrist rotation, is a passive movement which can be locked in various positions by a bolt. The socket is made to a cast of the stump and, in the case of short stumps, may be carried over the acromion for greater security. The usual harness has a three-point attachment to the socket, so that the arm is firmly suspended from the shoulder, and passes across the back in a figure-of-eight loop round the opposite axilla. An operating cord from the posterior aspect of the contralateral axilla passes to the arm in essentially the same manner as the below-elbow operating cord and is activated similarly. This cord passes through a loop in a lever attached to the proximal part of the forearm, thus permitting active elbow flexion. When the elbow is locked this same pull is transmitted through to the terminal device for its operation. The elbow locking thong is attached to the harness anteriorly over the top of the shoulder of the amputated side. 
A thrust of the stump into the socket together with extension at the shoulder creates a pull which trips the elbow locking mechanism.

The terminal device-The commonly used hands have been described (Figs. 1 and 2). There have been many attempts to design a versatile mechanical hand. The difficulty arises from the fact that the success of the natural hand as a prehension tool derives in a great part from its ability to take up the form and posture most appropriate for the task to be performed (Capener 1960). The intricate coordination between various muscles and muscle groups in association with the most delicate and comprehensive sensory feedback system makes this possible. It is

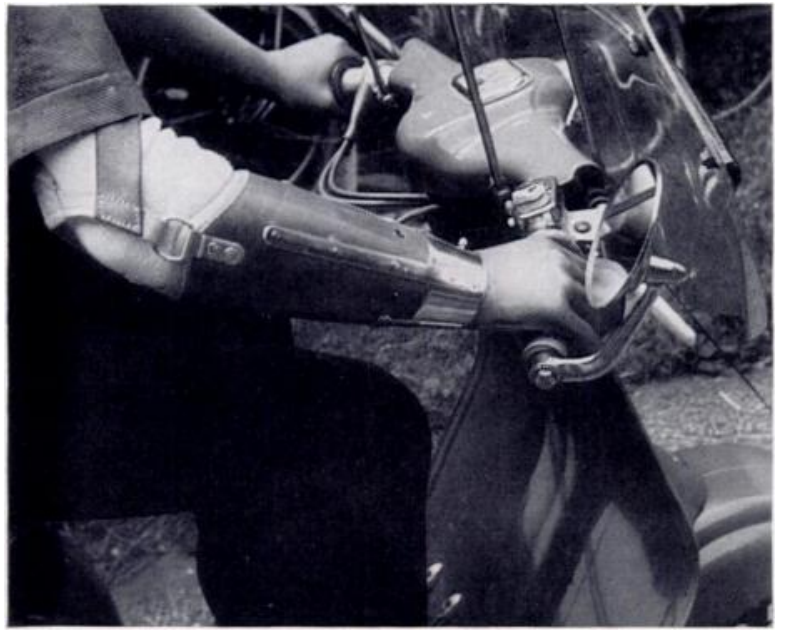

FIG. 1

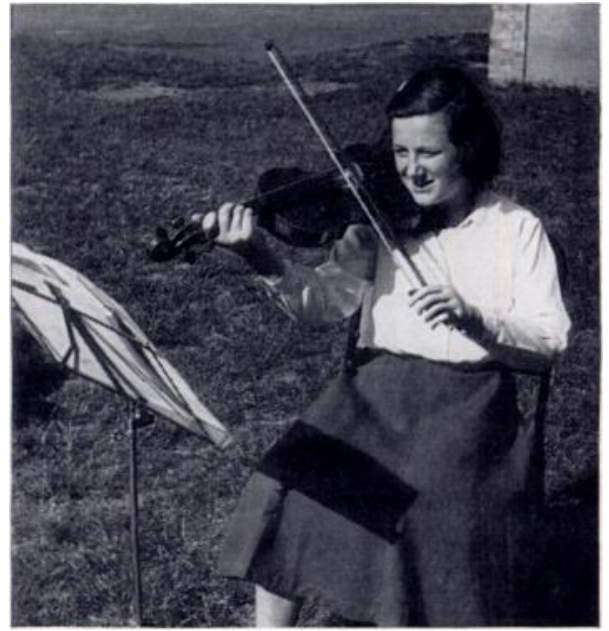

Fig. 2

The standard hand in use for riding a motor scooter and playing the violin.

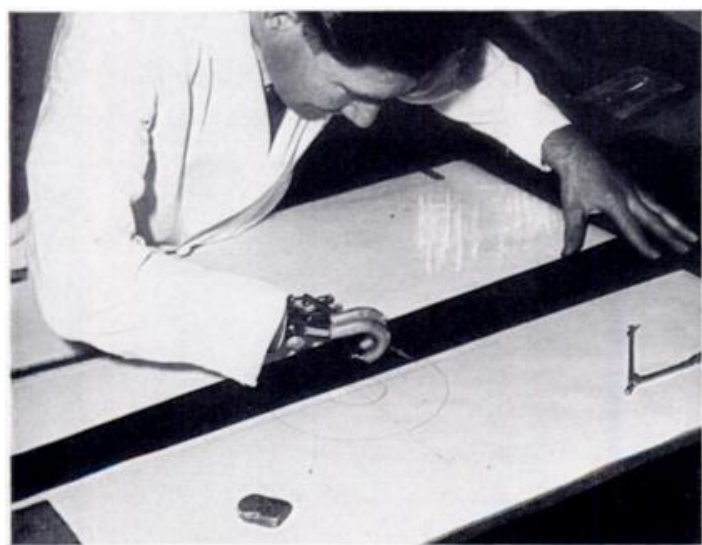

Fig. 3

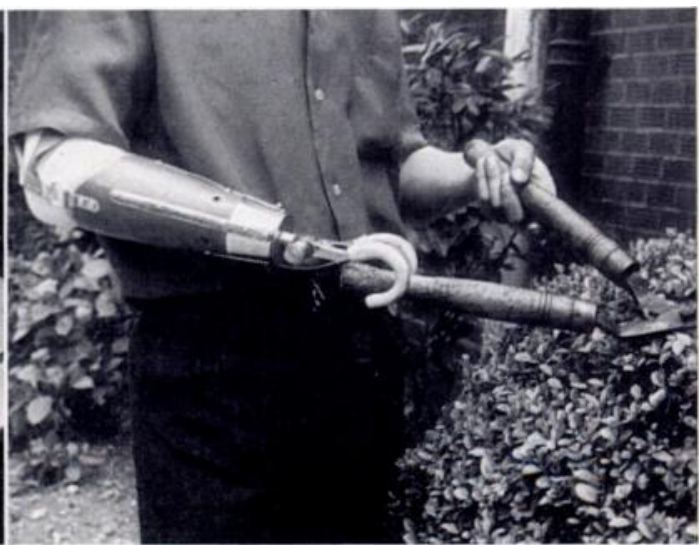

FIG. 4

The standard split hook being used for the simpler task of using the pencil while the good hand controls the set square (Fig. 3); and the split hook in use in the essentially symmetrical bilateral activity of hedge clipping.

not practicable to introduce more than two or three of the basic grasp postures in any one mechanical hand, so that the hand, for tasks which are inappropriate to these postures, becomes a clumsy tool. The split hook is therefore by a long way the most effective general purpose prehension device, while one admits its aesthetic shortcomings (Figs. 3 and 4). Most split hooks in use today are operated in such a way that a pull on the cord causes the hook to open, closure being by spring or elastic tension. This has been called the voluntary opening hook. The opposite concept, the voluntary closing hook, might seem to be attractive and likely to permit of more sensitive control of pressure, but there are serious disadvantages. It is arduous 
to maintain a grasp on an object with such a device because the shoulders and proximal part of the arm require to be held rigid to maintain a pull on the operating cord. A lock has therefore to be added which functions by following down the closure and preventing reopening when the pull is relaxed, and doing this in such a way that there is no backlash. This is difficult to do technically.

A number of other terminal devices are available (Figs. 5 and 6). These are mostly specialist tools for special tasks and commonly are kept as part of the user's tool kit.

Functional characteristics of the conventional prosthesis-The object of the prosthesis is to enable the wearer to carry out manual functions in as natural a way as is possible. One can consider the upper extremity as being composed of two parts, the prehension component, namely the hand, and the remainder of the arm, which, like the jib of a mechanical grab, serves to position and orientate the prehension device in space. The conventional prosthesis provides a terminal prehension device, which is under active voluntary control, and the means of positioning it in space. There are certain positions in space, for example behind the head, where, because of the position of the shoulder joint, the pull of the control cord cannot be brought to bear on the terminal device. Fortunately, providing grasp can be maintained, there are few tasks which require the hook to be opened in these positions.

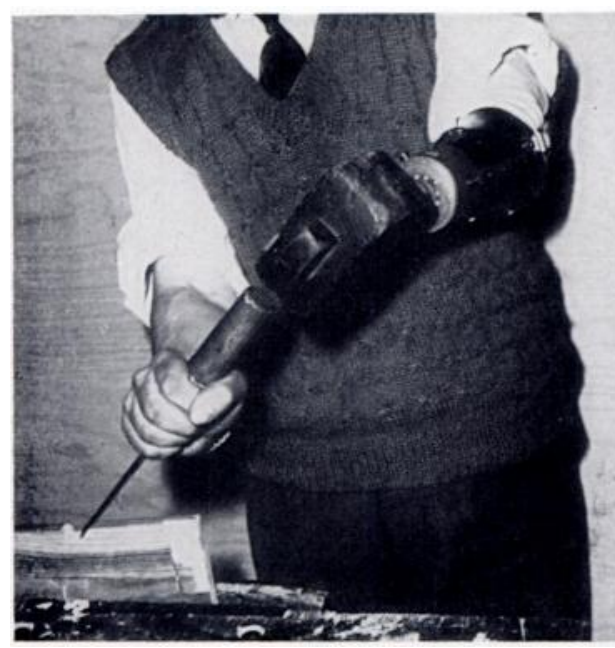

FIG. 5

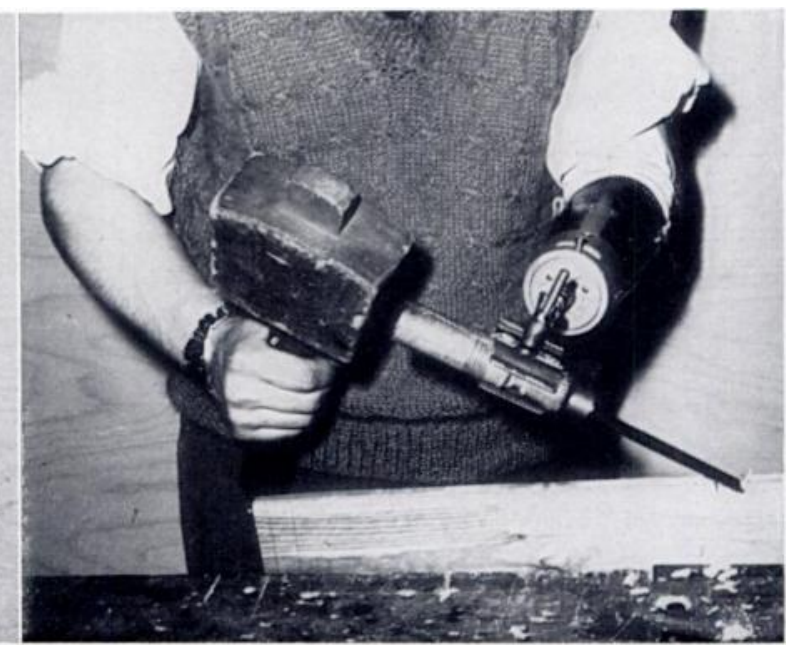

Fig. 6

In Figure 5 the chisel is held in the dominant normal right hand; but in Figure 6 the patient is left-handed and holds the chisel in the dominant artificial hand.

The forearm amputee can position his terminal device in space at will as he has his shoulder and elbow joints intact. It is more difficult for him to orientate his terminal device. He can preset the arm in the desired degree of pronation or supination by using the passive mechanism, and when the elbow is flexed he can create the effect of pronation by abduction at the shoulder. It has been found that the absence of wrist flexion and extension and of radial and ulnar deflection is of little practical importance. Figures 7 to 11 show various household activities being done with the conventional artificial limb.

Similar considerations apply to the above-elbow subject who in addition has to control the position of his artificial elbow joint. Elbow flexion by the cord from the harness together with the lock control enable him to pre-position the terminal device at the desired level. The wearer is unable to flex the elbow and activate the hook simultaneously unless the controls for these two movements are separated. This can be done by different harnessing technique, particularly that devised by Hepp and Kuhn (1960) in Münster. In general it has not been found necessary for most people's purposes to do this.

vol. 47 B, No. 3, AUGUSt 1965 


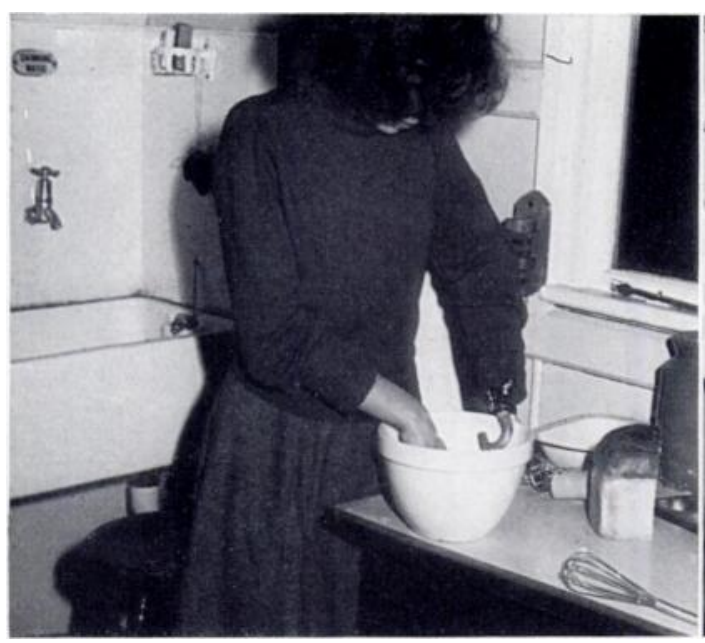

FIG. 7

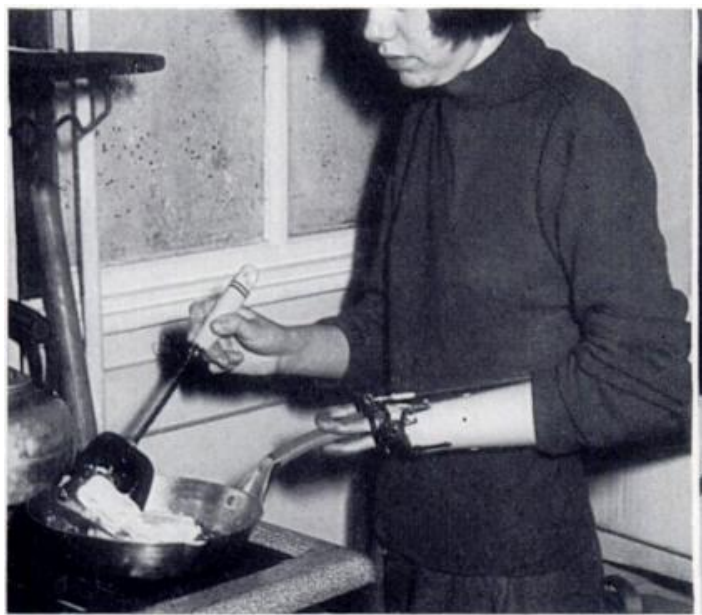

FIG. 9

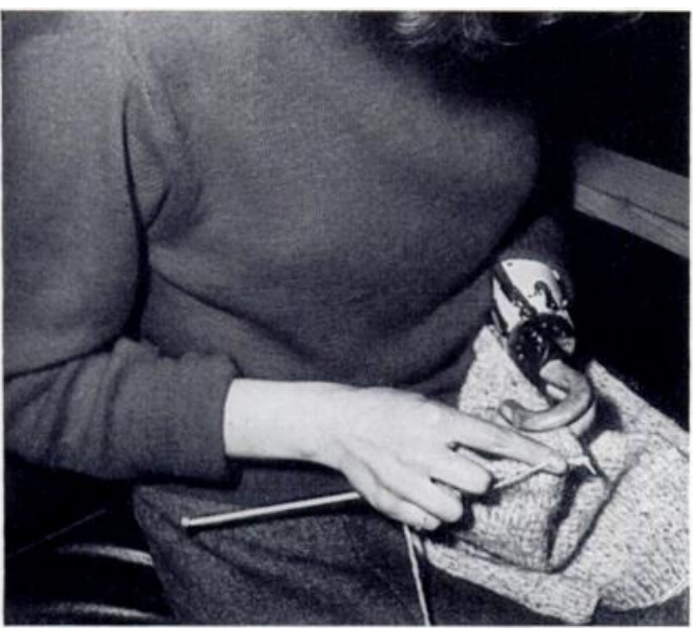

FIG. 11

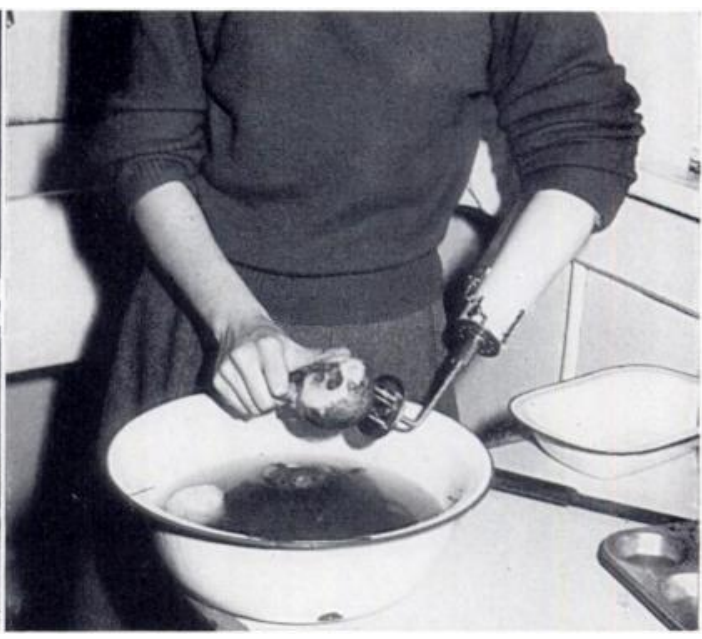

FIG. 8

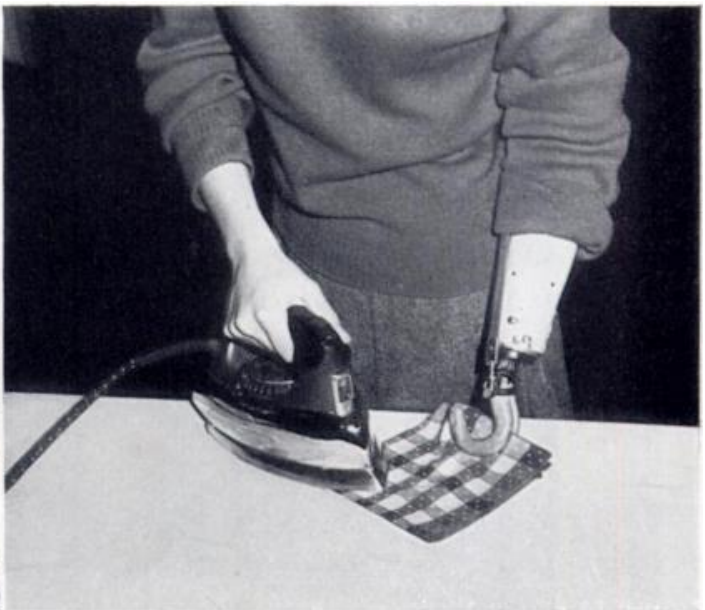

FIG. 10

Figs. 7 To 11

Everyday household duties are easily done by the housewife using the ordinary split hook or simple forms of terminal devices. Because most household duties need bimanual skill the housewife is very little handicapped when she uses the conventional artificial limb. 
These devices are simple, robust and relatively free from mechanical troubles, the utmost reliability being important to all and vital to the bilateral amputee. With their aid there are very few occupations which are beyond the capabilities of a patient with amputation of one arm, particularly if he has retained his own elbow joint. The bilateral amputee, even if both stumps are above the elbow, can become socially independent and can earn his living. One such subject is an artist of considerable merit, another is a wood-turner and yet another is a solicitor's clerk. The latter two drive their own cars, and all enjoy hobbies of various kinds. The average limb wearer develops surprisingly delicate control of the movements of his prosthesis. For example, many amputees have discovered a trick which they can do and a normal person cannot-picking up a piece of cigarette ash in the hook without crushing it. This is done under visual control and there is no doubt that much of the sensory feedback in relation to what is happening with the prosthesis is visual. Nevertheless, true sensory feedback does accrue through the reactionary pressures of the socket and of the harness. For example, all amputees know without looking how far the hook is open within fairly fine limits and know how firmly they are grasping an object. Thus, an anatomist with an aboveelbow amputation can feel the thickness of a cutaneous nerve when it is picked up in forceps held in the hook. I will never forget the amazement of a distinguished visiting orthopaedic surgeon who, with gallant contempt for personal danger, permitted a demonstrator to grasp his nose in his split hook and felt the pressure more of a caress than the crushing pincers that he had anticipated.

Even a blind bilateral amputee derives useful sensation from these sources. For example, one such patient is able to serve behind the counter in his tobacconist shop.

It will be seen therefore that the potential function available to users of conventional artificial arms is considerable. Nevertheless, it has to be admitted that a number of unilateral amputees either reject the arm entirely or prefer to use a passive prosthesis for purely cosmetic reasons. The explanation of this is probably that the average person can attend to all his daily needs and moreover be little handicapped in his occupation with one good arm, possibly aided by using the other axilla or the crook of the elbow for holding objects or the stump for holding objects down. The functional use of the prosthesis is a learned skill, and until this skill is acquired the prosthesis may be more of a burden than of a help. It follows, especially when the patient has had sufficient time after his amputation to develop a one-handed pattern of existence, that those without any great incentive tend to give up the attempt. Nevertheless the high skill attainable by those who do persevere and particularly by bilateral amputees, whose greater helplessness gives them the greater incentive to use the prosthesis, provide a yardstick against which new developments such as powered prostheses may be compared.

Even the unilateral through-shoulder amputee can achieve a certain degree of function, although it has to be admitted that the forces and excursion that can be imparted to the control cables are reduced. Fortunately, bilateral amputation at this level is a great rarity. Nevertheless there has always been a steady small incidence of children born with bilateral amelia. Several such patients have been fitted with conventional arms, notably one who was able to control three separate functions of the prosthesis on each side. It has to be admitted that the bodily movements required to achieve some of these movements, particularly at the extremes of range, were excessive. Nevertheless, once the arms were on, this boy became virtually socially independent.

To summarise, the advantages of conventional artificial arms might be said to be simplicity, reliability and freedom from maintenance problems, robust versatile function, sensory information direct to the central nervous system, ample muscle power and range of movement for unobtrusive and silent operation of the prosthesis, social independence and a practically unlimited choice of occupation for all single amputees and many with bilateral amputations. Disadvantages are that the shoulder harness is irksome and is unsightly 
particularly for women, the control cord cannot be operated in certain postures, and the high-level amputee who requires to be able to control a greater number of movements tends to have a fewer number of available body sites for harnessing and an insufficient range of movement and power.

\section{POWERED LIMBS FOR CHILDREN}

The application of external power to artificial limbs was already being studied in this country when it became apparent that a number of children born with major congenital defects as a result of thalidomide would require prostheses. We had adopted several general principles. 1) On the assumption that the conclusions given above in relation to the conventional arms are valid, it was considered that those most likely to benefit from powered prostheses were the bilateral high-level amputees. 2) It is important to acceptance and tolerance of the prostheses over the years that the wearer should integrate them into his body image. The prostheses should therefore conform in appearance and in movement patterns as nearly as possible to natural arms. Robot-like timing and unnatural angular movements should be avoided. Partly for the same reason, both sides should be fitted and bimanual function preserved. 3) The control system should enable the wearer to manipulate the prostheses without thinking. The speed, force and excursion of each movement should be controllable, and simultaneous performance of two or more movements is desirable. Sequential controls are therefore contra-indicated. 4) The introduction of a powered actuator would reduce or even cut off the sensory information arising distal to the actuator. Means of sensory feedback should therefore be sought. 5) Pneumatic actuators had been developed with valves for their control (Kinnier Wilson 1962). Work was also proceeding on a myo-electric system.

If these premises were correct we felt that the children with bilateral amelia and very short phocomelia should be benefited by externally powered arms (Figs. 12 to 14). We had fitted conventional prostheses to children with similar defects, and although the requisite movements could be performed and the children attained a degree of independence, the actuation of the arms was laborious and involved considerable contortions. Most of these children had normal or near normal legs and had developed immense dexterity, using their feet in lieu of hands. Most had come to us at about the age of leaving school when they became self-conscious about this. Although the prostheses may have tided these patients through the crisis of facing up to the world, most have discarded the prosthesis sooner or later and reverted to using the feet.

It seemed, therefore, that if this new group of young patients was to be helped they should be fitted with prostheses early enough for them to form their lives with an ingrained pattern of prosthetic use. The normal child moves its arms from birth, first merely moving them about largely from the shoulder but soon holding large objects between the hands in the so-called pat-a-cake grasp. Some form of prostheses should probably be fitted even as early as three months to ensure integration in the developmental pattern. It is probably impossible to teach so young a child voluntary control of powered arms, but simple, soft, unjointed arms can be applied so that movements of the shoulder girdle impart some movement into the arms and the child may even attempt pat-a-cake grasp. At the least, the child is given the opportunity to enter the dawn of conscious experience with this prosthesis as part of his body image.

The attempt to apply more functional arms can probably first be made at about the time the child attains independent sitting balance. However, the body movements normally harnessed to control conventional arms represent a learned skill. We doubted whether children of this age could be taught such skill. And so it proved in the event.

Following the practice of Marquardt (1964) we first applied a system providing the equivalent of synchronised medial rotation at the shoulders to give pat-a-cake grasp. Marquardt so arranged the control valves that rounding the shoulders (a body movement used commonly in prosthetics) caused medial rotation and the reverse body movement caused lateral rotation. In our hands even two-year-old children failed to learn this movement. I understand that 
similar difficulties were experienced at Oxford, where the first pat-a-cake arm was fitted in Britain. However, when it was arranged that forward trunk bending (as would occur naturally when a child saw something interesting in front of it) caused the medial rotation, and lateral rotation resulted when the child lost interest and extended the trunk, the result was dramatic. The children have learned to perform the movement purposefully within minutes or at the most within a couple of days, and no child so equipped has failed to learn the control.

Thus a further principle can be postulated. The body movement for a control should be natural to, or synergic with, the prosthetic movement to be produced. It seems logical to extend this principle to subjects of an educable age to conform with the third of the principles enumerated above. It seems also that crossed controls using contralateral movements should be avoided if possible.

Unfortunately, by the time components were available and we had appreciated this principle, all the thalidomide children were rather older than one would have wished, the

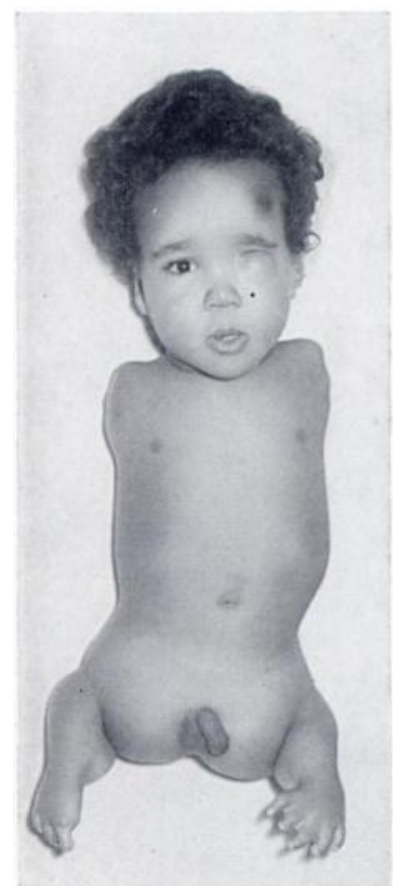

FIG. 12

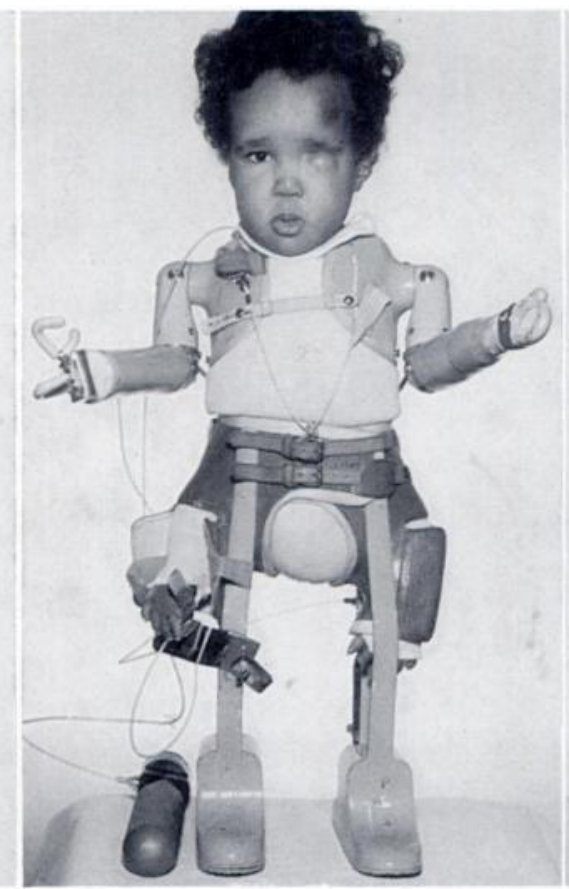

Fig. 13

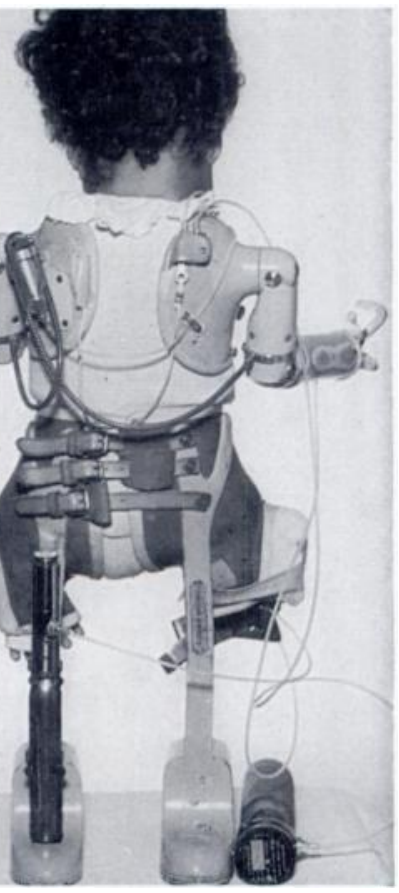

Fig. 14

A child with an upper extremity amelia (Fig. 12) has been equipped with a pneumatic powered pat-a-cake hand on the left and a pneumatic power-operated hook on the right. The control cords for the pat-a-cake grasp are shown in front and behind (Figs. 13 and 14). The hook is controlled by the toes of the right foot.

youngest we have fitted being twelve and a half months. In view of the ready acceptance, however, we believe it would be worth making the first attempt when the child achieves sitting balance at about six to eight months (Illingworth 1964).

The children appear to derive pleasure from this simple form of grasp and to use it freely and naturally to extend their play activities. It must help them to acquire experience and develop. Sooner or later, within a week or two in the older and more intelligent children, the crude pat-a-cake grasp ceases to give satisfaction and the child loses interest or shows outbursts of temper when frustrated by a task too complex for the pat-a-cake. It becomes imperative to introduce a more sophisticated grasp.

A pneumatic powered hook can now replace the passive hand on one side and, when its control is mastered, on the other. It is probably best to disconnect the upper arm rotator 
from the dominant arm at this stage, retaining it on the other side so that bimanual function of the spoon and pusher type can be learned. If phocomelic digits are present it is logical to use them to control the valve for the hook. This has been confirmed by the facility with which children so fitted learn the control. Those with complete amelia take longer to learn, but forward movement of the shoulder to close the hook and the reverse to open it seem the most synergic available movements and have been learned fairly consistently with training. We have used the toes to control the valves for the hooks for some children whose phocomelic feet are useless for walking, on the analogy that children with normal legs and absent arms naturally use their feet as organs of prehension. It is interesting that these children learned this control readily.

At this stage it becomes apparent that the child needs to move the prehension device in space actively rather than by passive pre-positioning as hitherto. Elbow flexion seems to be the most useful active movement for this purpose but the forces required are comparatively

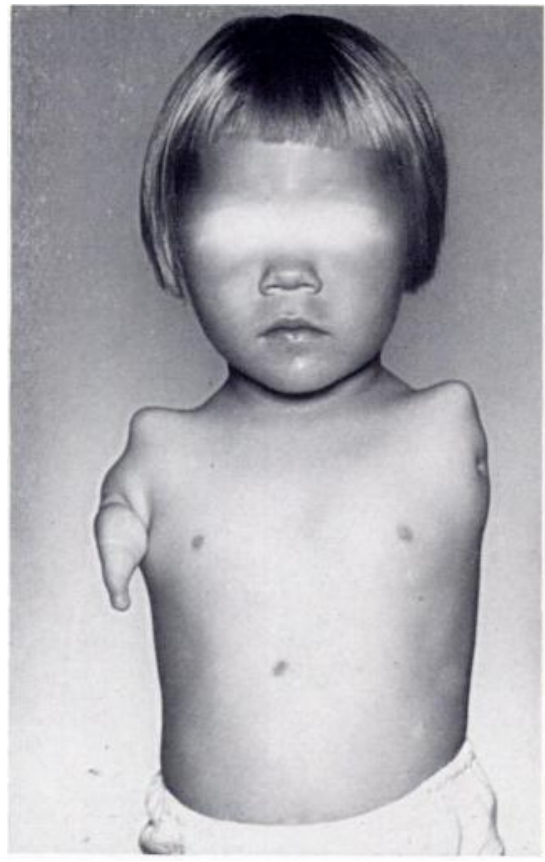

FIG. 15

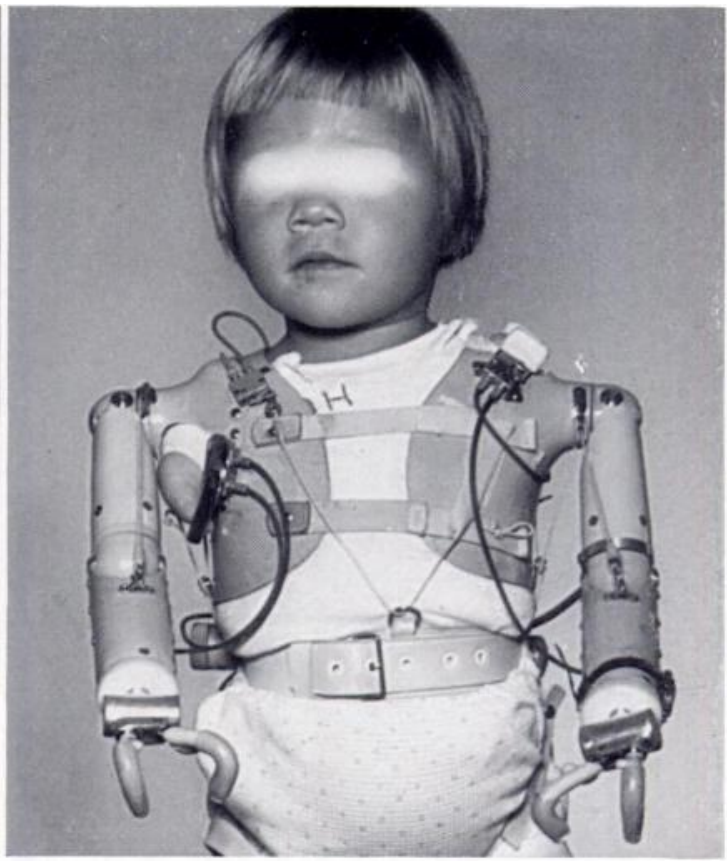

Fig. 16

This child has been equipped with pneumatic-powered internal rotation of the left arm above the elbow, which is controlled by bending the trunk forwards or backwards; with a powered hook on the left controlled by forward or backward movement of the acromion, with a powered hook on the right controlled by the photometric digit; with elbow flexion on both sides controlled by the direct body pull of lateral trunk bending.

large, so the drain on the power storage system would be heavy. We harnessed lateral trunk bending towards the contralateral side together with shoulder elevation to give a direct bodypowered lift to the forearm (Figs. 15 and 16). This is a completely natural movement and most children have learned the control at first attempt and acquire great precision and sensitivity. Ample power and excursion are available and the movement is not obtrusive. We have felt it advisable to introduce only one additional movement at a time, but children as young as two years have reached this stage. With this equipment and a self-levelling spoon the child can learn to feed himself, and a wide variety of games and activities opens up for him (Figs. 17 to 24).

He needs the addition of active pronation and supination to feed with normal utensils, and for many other activities. This can be introduced either as a separate movement or mechanically linked to elbow flexion. The latter technique has been adopted by McLaurin 
(1964) and may be an essential for amelic children because of the few available control sites. Simpson (1964) and Kiessling (1964) use flexion and extension of the wrist linked to elbow flexion for a somewhat similar purpose, but the movement is perhaps less aesthetic. We have now fitted a simple pronator-supinator controlled by forward and backward movements of the acromion, which can be operated simultaneously with the body-controlled elbow flexion, allowing a natural feeding action.

With this system of elbow flexion by body pull, and extension by gravity, a means of locking the elbow is required for such things as writing to provide a resistance to passive flexion. A lock has now been designed which uses the first fraction of the travel of the elbow flexion cord to disengage the lock, and continued pull to cause the elbow to flex. Sudden relaxation allows the lock to engage while controlled relaxation allows extension to take placea somewhat similar principle to that used in some roller blinds.

To avoid confusing the child we endeavour to anticipate the ultimate equipment so that the appropriate control for each movement may be introduced from the start, and he does not have to relearn the association of a given control movement with different prosthetic responses. We also recognise that any failure of the prosthesis to respond or any inadvertent response to casual body movements will confuse the child and undermine the pattern of learning.

It is important not to overrate the value of powered arms. A child with phocomelic arms which are long enough for him to reach his mouth and to approximate them in front of the body, and who uses hands in preference to his feet, is likely to be more effective with his natural hands than with any artificial arms yet devised.

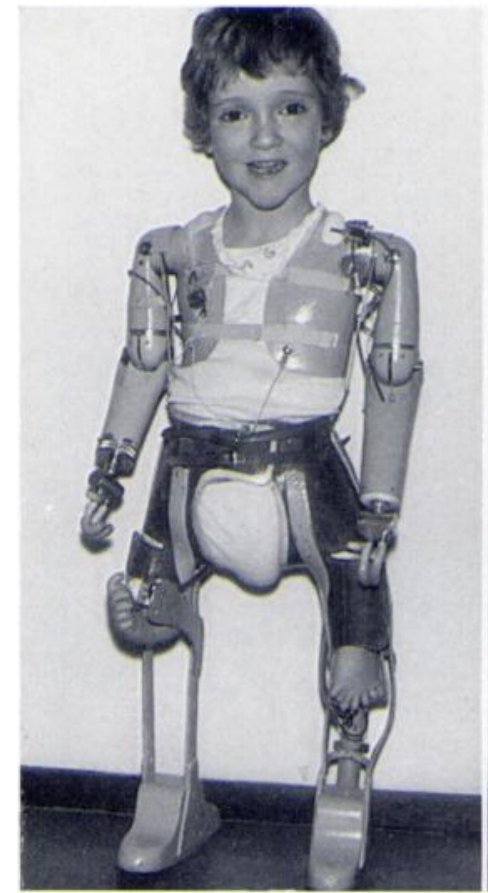

FIG. 17

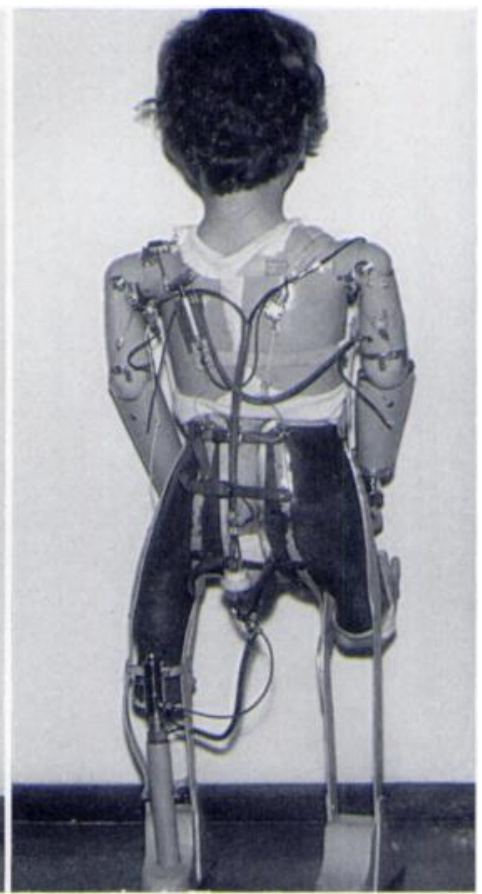

FIG. 18

This child with bilateral upper limb amelia has been equipped with pneumaticpowered internal rotation of the upper right arm controlled in the same manner as the pat-a-cake movement; with powered hooks controlled by the toes; with elbow flexion controlled by direct pull; with automatic elbow locks concealed in the elbows; and with powered supination and pronation of the left and dominant arm controlled by the acromion.

\section{ADULT PNEUMATIC ARMS}

Work on components for adult pneumatic arms has had to take second place to the urgent needs of the children, and it is only now becoming possible to resume trials on adult patients. Nevertheless, the work with children has established some principles and provided a number of pointers as to how future work should be directed.

With the children the need was so urgent that we had to work empirically. Thus we tended to provide a device which would give the minimum of function, and to add other degrees of freedom one by one as the need became apparent in relation to the child's developing activities. There is a need for a study of the angular movements and accelerations of the various 


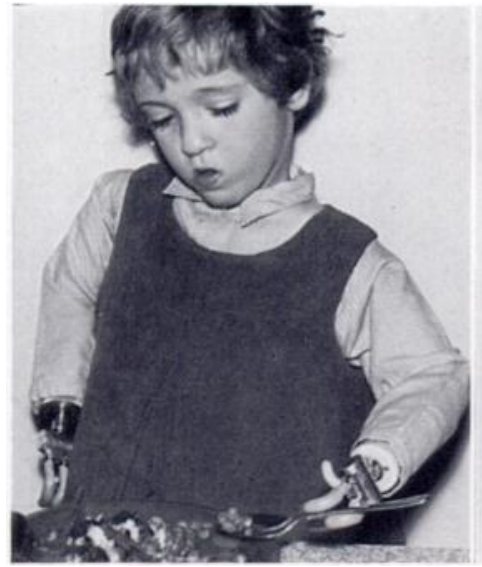

FIG. 19

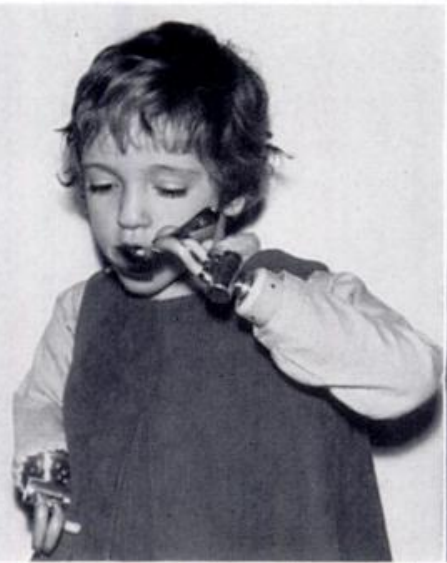

FIG. 20

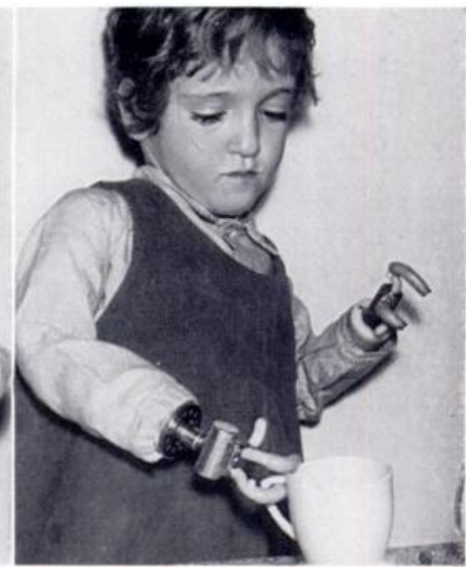

FIG. 21

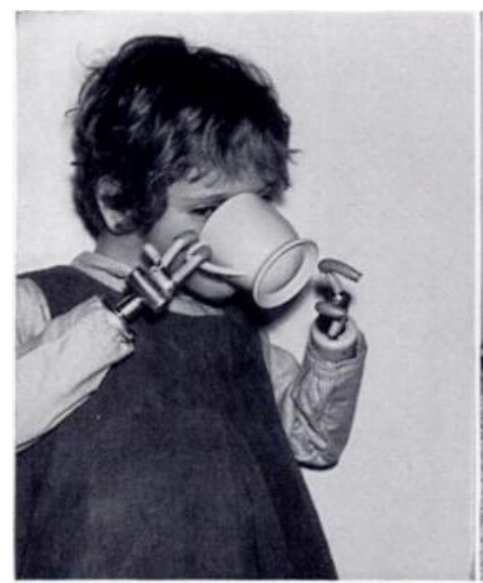

FIG. 22

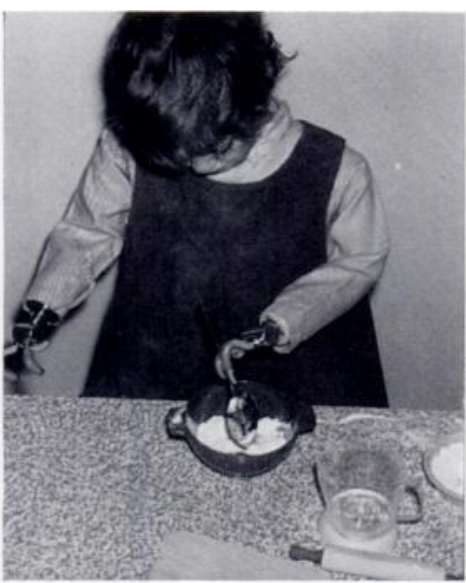

FIG. 23

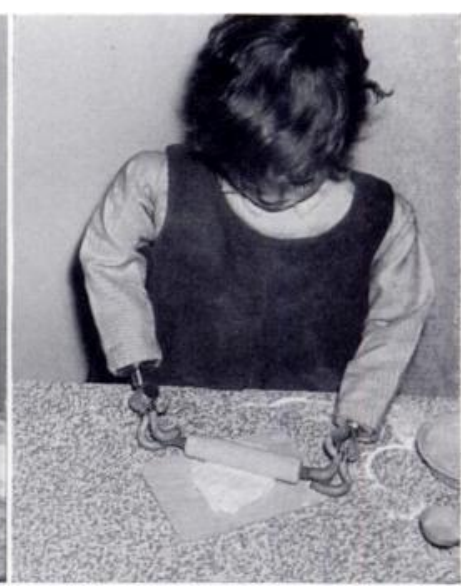

FIG. 24

The same child as in Figures 17 and 18. She can eat-and note how she keeps the spoon level by the powered supination-and drink; she plays well and, because of the above elbow rotation, she uses the prostheses in bilateral activities.

components of the normal arm during all the tasks of daily living, and a similar study of the modes of grasp for the various tasks. It would then be necessary to interpret these in relation to known prosthetic possibilities and to determine how these movement patterns could be simplified. A systematic plan could then be devised in terms of the specific movement to be powered, of the possibility of linking two or more movements for given tasks or indeed the need for more elaborate programming.

Assuming that we were correct in our belief that powered devices would be useful mainly for those with bilateral high level amputations or the equivalent, we have thought that the prime movements required would be grasp, wrist rotation (pronation and supination) and elbow flexion. It is already apparent from the work on children that more than this is required. The equivalent of medial rotation at the shoulder on at least one side is necessary if true bimanual function is to be provided, and I believe this to be not only possible but desirable.

A patient with a prosthesis for disarticulation at the shoulder cannot reach forward without active flexion at the shoulder. This is not so vital to a patient who is performing a task when he is standing, because he can move the terminal device forwards or backwards in space by shuffling on his feet or by swaying forwards and backwards. It assumes greater importance when the subject is seated working at a table, when the extent of forward and backward movement which can be created by swaying forwards from the hips is more limited, 
particularly when the lower limbs also are absent or when the hip joints are non-existent as in some children affected by thalidomide. It seems therefore that it will also be necessary to power the movement of flexion at the shoulder, at least for some patients. Abduction at the shoulder may also be required for working when seated at a table.

It is probable that some flexion and extension of the wrist or radial and ulnar deflection at the wrist, or both, possibly linked to pronation and supination, may be advantageous particularly for feeding and for toilet self care.

It will be apparent that as one strives to enhance the function of devices such as these, more and more control sites on the body have to be found for the added movements. Marquardt's (1962) view is that one side only should be fitted with a functional arm the control of which is taken from control sites on both sides of the body; a merely passive arm, if anything at all, is fitted on the contralateral side. Simpson (1964) takes the same view. As mentioned previously, I personally am against crossing the mid-line in search of control sites unless there is no alternative, and I believe that although one arm may be built up as the master arm and contain a number of degrees of freedom, the other arm should still remain a functional device even if it only has an active means of prehension, with the ability to pre-position the terminal device in space passively. Assuming the optimum number of degrees of freedom in the master arm, every additional degree of freedom which can be added to the second arm will enhance the total function.

Sensation becomes the next problem because it is only from sensory information that the wearer can monitor the performance of his prosthesis. I have shown earlier that the wearer of a conventional prosthesis derives sensory information from his harness, and that the intervention of a powered actuator in the linkage between the control site and the prosthesis reduces or eliminates this sensory channel. This is particularly the case with pneumatic actuators which are relatively vibration-free. Thus the only sensory information available to the wearer is visual, though he possibly also gets some information from the sound of the exhaust gases. It is probably more important to provide direct sensory information from the grasping device than from any of the other movements. It is therefore worth considering whether or not a hybrid prosthesis might provide a compromise. With this the hook or hand would be operated by a cable from a direct body harnessing while the remainder of the movements would be power actuated. The problem of sensory feedback from the prosthesis remains to be solved and the development of sophisticated control systems which will be satisfying to the user depends on its solution.

The operation of multiple controls will be much easier for those with phocomelic hands, for whom it is possible to lay out the control switches or valves in the form of a keyboard which the user can play like a piano. This has already been achieved by a young man of twenty-seven with bilateral phocomelia who is trying out pneumatic prostheses providing three degrees of freedom on each side. He had worn conventional prostheses for eleven years and his immediate reaction to the powered limbs was delight in the enhanced range of activities available to him, the increased power of grasp and the saving of energy expenditure. The arms are not yet completed and it remains to be seen how the arms function under field conditions.

Elsewhere in this issue Kinnier Wilson discusses some of the technical requirements of pneumatic actuators. Much work remains to be done on the design of equipment. For example, although a working pressure of 70 p.s.i. was chosen rather arbitrarily, evidence is now accumulating that higher pressures, perhaps in the region of 240 p.s.i., might have advantages. These and many other considerations are being studied.

\section{MYO-ELECTRIC DEVICES}

Finally a word about myo-electric prostheses (Kobrinski 1960, Bottomley 1964, Bottomley and Cowell 1964) in relation to the amelic children and to high level bilateral amputees.

VOL. 47 B, NO. 3, AUGUST 1965 
Before these can be applied we will have to discover whether such individuals can produce myo-electric signals at will from remaining muscle groups in such a way that they can be isolated and collected. The muscles used must not produce signals during ordinary daily events, such as moving the head, walking, coughing, etc. Ideally, they should be muscles producing a body movement synergic with and natural to the prosthetic movement to be controlled. Perhaps electrodes in the form of implants giving a telemetered signal may prove a possibility.

\section{SUMMARY}

I have tried to describe the functional attainments of amputees using conventional prostheses. I have outlined the work we have been doing on pneumatic arms for infants and children. I have discussed some of the practical problems in applying pneumatic and myo-electric systems to adults. The inescapable conclusion that one must reach is that two basic clinical requirements must be met before really significant advance is made in this field. Firstly, we must find a method of providing sensory information from the prosthesis. Secondly, we must find a way of utilising more control sites. So far we have only been able to make use of direct movements or myo-electric signals. In the high level bilateral subjects more controls are required than appear to be available. Capener has suggested, in more than one discussion that I can recall, the possibility of using the voice and I have no doubt that a subject could be trained to reserve certain frequencies for prosthetic controls. It may be that we will have to think along some such lines as this.

I am indebted to Sir George Godber, Chief Medical Officer, Ministry of Health. for permission to contribute; to Dr I. Fletcher and Dr R. Redhead for their clinical help; to Miss L. B. E. Galloway whose skill, patience and ingenuity have led to much of our progress; and to Mr E. B. Ferrill for many of the photographs. Crown copyright reserved.

\section{REFERENCES}

Botromley, A. H. (1964): The Control of Muscles. Progress in Biocybernetics, 1, 124.

Bottomley, A. H., and Cowell, T. K. (1964): An Artificial Hand Controlled by Nerves. New Scientist, $21,668$.

CAPener. N. (1960): Prosthetics International. Proceedings of the Second International Prosthetics Course, Copenhagen, 21.

Hepp, O., and Kunn, G. G. (1960): Prosthetics International. Proceedings of the Second International Prosthetics Course, Copenhagen, 133.

Illingworth, R. S. (1964): Normal Development. British Medical Journal, ii, 1,245.

KiesSLING, E. A. (1964): Personal communication.

Kobrinski, A. E. (1960): Problems of Bioelectric Control. Automatic and Remote Control. Proceedings of the First International Congress of International Federation of Automatic Control. Moscow, 619.

Mclaurin, C. A. (1964): Personal communication.

Marquardt, E. (1962): Le Bras Pneumatique. Proc Cours Internationale de Prosthese, Quatrieme Session, 1961-Imprimerie Nationale Paris.

Marquardt, E. (1964): Providing Armless Babies with Active Prostheses in 2nd Year of Life. Inter Clinic Bulletin, III, No. 4, 3.

Simpson, D. C. (1964): The Development and Control of a Powered Prosthesis for Children. Scottish Home and Health Department Bulletin, 22, 67.

Wilson, A. B. Kinnier (1962): Technical Report of the National Fund for Research into Poliomyelitis and Other Crippling Diseases, pp. 5-7. 